\title{
Antiphase ordering and surface phases in lithium aluminate
}

Richard R. Vanfleet

rrv3@byu.edu
J. A. Simmons
D. W. Hill

M. M. C. Chou

B. H. Chai

Follow this and additional works at: https://scholarsarchive.byu.edu/facpub

Part of the Astrophysics and Astronomy Commons, and the Physics Commons

\section{Original Publication Citation}

Vanfleet, R. R., J. A. Simmons, D. W. Hill, M. M. C. Chou, and B. H. Chai."Antiphase ordering and surface phases in lithium aluminate." Journal of Applied Physics 14 (28).

\section{BYU ScholarsArchive Citation}

Vanfleet, Richard R.; Simmons, J. A.; Hill, D. W.; Chou, M. M. C.; and Chai, B. H., "Antiphase ordering and surface phases in lithium aluminate" (2008). Faculty Publications. 152.

https://scholarsarchive.byu.edu/facpub/152 accepted for inclusion in Faculty Publications by an authorized administrator of BYU ScholarsArchive. For more information, please contact ellen_amatangelo@byu.edu. 


\title{
Antiphase ordering and surface phases in lithium aluminate
}

\author{
R. R. Vanfleet, ${ }^{1, a)}$ J. A. Simmons, ${ }^{2}$ D. W. Hill, ${ }^{3}$ M. M. C. Chou, ${ }^{3, b)}$ and B. H. Chai ${ }^{3}$ \\ ${ }^{1}$ Department of Physics and Astronomy, Brigham Young University, Provo, Utah 84602, USA \\ ${ }^{2}$ Advanced Materials Processing and Analysis Center (AMPAC) and Department of Physics, \\ University of Central Florida, Orlando, Florida 32816, USA \\ ${ }^{3}$ Crystal Photonics Inc., 5525 Benchmark Lane, Sanford, Florida 32773, USA
}

(Received 16 June 2008; accepted 24 September 2008; published online 12 November 2008)

\begin{abstract}
Antiphase domains are seen in single crystal gamma lithium aluminate $\left(\gamma-\mathrm{LiAlO}_{2}\right)$ with $16.7 \mathrm{~nm}$ periodicity in the $\langle 110\rangle$ direction. Alternate domains have a $\frac{1}{2}$ [001] shift. Beta phase lithium aluminate $\left(\beta-\mathrm{LiAlO}_{2}\right)$ is seen to form on the surface of the as-received wafers with an epitaxial strain limited relationship with the bulk $\gamma$ phase. The orthorhombic $\beta$ phase aligns with the $a$ and $b$ axes $(0.528$ and $0.630 \mathrm{~nm})$ matching with the tetragonal $\gamma$ phase's $a$ and $c$ axes $(0.5168$ and 0.6268 $\mathrm{nm})$. The $\gamma$ and $\beta$ phases are seen to have different etch rates. The $\beta$ phase converts back to the $\gamma$ phase above $450{ }^{\circ} \mathrm{C}$. (C) 2008 American Institute of Physics. [DOI: 10.1063/1.3014193]
\end{abstract}

\section{INTRODUCTION}

Gamma phase lithium aluminate $\left(\gamma-\mathrm{LiAlO}_{2}\right)(\gamma$-LAO) is a near lattice match substrate for the $m$-plane $\mathrm{GaN}(1 \overline{1} 00)$. $\gamma$-LAO has a tetragonal lattice structure $\left(P 4{ }_{1} 2{ }_{1} 2\right)$ with lattice constants of $a=0.5168 \mathrm{~nm}$ and $c=0.6268 \mathrm{~nm}{ }^{1}$ The (100) surface not only has a small lattice mismatch with GaN, but has an arrangement of atoms that matches the (1010) face of $\mathrm{GaN}$. Work with LAO focused on the growth of GaN films with little attention paid on the substrate material itself. In this manuscript we focus on lithium aluminate.

Gamma phase lithium aluminate was originally discussed in the literature by Bertaut et $a l^{2}$ and the structure was clarified by Marezio. ${ }^{1}$ Cockayne and Lent ${ }^{3}$ then produced large single crystals of the $\gamma$ phase by the Czochralski technique. It was used as a substrate for GaN growth by Hellman et al. ${ }^{4}$ in 1997 where the resulting growth orientation was not the $m$-plane as had been suggested. It was in 1999 that $\mathrm{Xu}$ et al..$^{5}$ and Waltereit et al. ${ }^{6,7}$ achieved the $m$-plane GaN orientation as had been postulated. There have now been many additional efforts in growing $\mathrm{GaN}$ on $\gamma$ phase lithium aluminate. ${ }^{8-16}$ There also have been some additional efforts in growing $\mathrm{ZnO}$ on this substrate. ${ }^{17}$

Previous work on LAO has seen a common morphology. GaN films on LAO show a stripe, corrugated, or "slate" morphology. ${ }^{6,11,12,18}$ This morphology has ridges running along the $\operatorname{GaN}\langle 11 \overline{2} 0\rangle$ direction. These ridges have shown to be replicas of the substrate, which also shows ridges running along the LAO [001] direction. This effect, while very common, is occasionally absent. This absence is attributed to a "better polishing procedure." ${ }^{10}$ Sun et al. ${ }^{11,18}$ have also shown that the corrugations of the as-received $\operatorname{LAO}(100)$ surface will significantly increase upon heating to $750{ }^{\circ} \mathrm{C}$ in vacuum. They have interpreted this increase in corrugations as decomposition of the LAO. They also suggested that there

\footnotetext{
a) Author to whom correspondence should be addressed. Tel.: 801-422-1702 Electronic mail: rrv3@physics.byu.edu.

${ }^{b}$ Present address: Department of Materials Science and Optoelectronic Engineering, National Sun Yat-Sen University, Kaohsiung, 80424, Taiwan.
}

are faces A and B of LAO (100) where an $\mathrm{HCl}$ etch produces visible roughening on one face and not on the other. ${ }^{11,18}$ While they confine their work on the smoother face A, they still see corrugations.

Although our own work began by studying defects in the $m$-plane GaN films grown on LAO, our interest focused on the LAO substrate itself. We observed stripe and crosshatched patterns in the imaging and superlattice peaks in the diffraction. These patterns indicate an antiphase domain structure in the $\gamma$ phase LAO. The anomalous etching of a different batch of wafers, where an $\mathrm{HCl}$ acid etch produced an opaque wafer similar to the description of Sun et al., ${ }^{18}$ prodded us to also look closer at the surface of this interesting substrate material.

We studied as-received wafers, wafers that have been at growth temperatures, wafers before and after etch, and a specific series of thermally treated wafers. Lithium aluminate wafers were supplied by Crystal Photonics, Inc. The Czochralski grown boule was commercially cut and polished producing $2 \mathrm{in}$. wafers with the [100] direction normal to the wafer surface. The wafers were subjected to various etch, thermal treatments, and growth conditions. Transmission electron microscope (TEM) samples were prepared using the focused ion beam (FIB) (FEI 200 TEM) at the University of Central Florida (UCF) with the ex situ and in situ lift out methods. Samples were investigated on FEI Tecnai microscopes at UCF and Brigham Young University.

\section{BULK LAO}

All lithium aluminate wafers studied exhibited common structural and diffraction characteristics. When viewed in the [001] direction, bright field and dark field TEM images show parallel lines (many, but not all overlapping) along the $\langle 110\rangle$ direction as seen in Fig. 1. Similar lines have been seen by Chou et al. ${ }^{19}$ Figure 1 shows a FIB prepared sample of an etched wafer. The surface regions A and B will be discussed later. It is regions $\mathrm{B}$ and $\mathrm{C}$ that show the parallel lines mentioned here. These lines have a repeat distance of approximately $17 \mathrm{~nm}$. The electron diffraction pattern shown in Fig. 


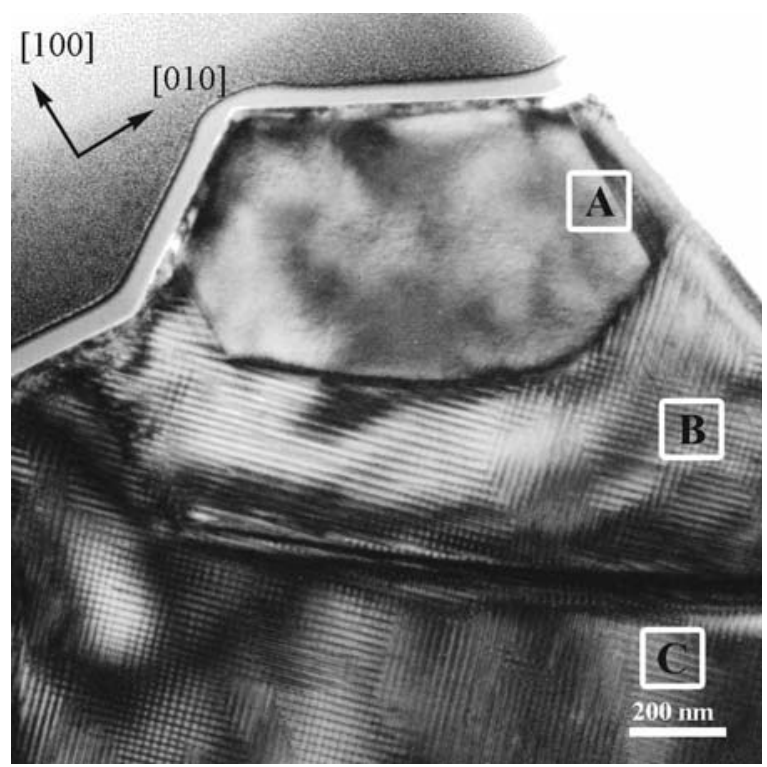

FIG. 1. Typical TEM bright field image of an etched LAO wafer in the [001] direction. Labeled regions (A, B, and C) show the surface with $\beta$ phase mesas, transitional region mixing typical $\gamma$ phase imaging with evidence of strain, and typical $\gamma$ phase material with regularly spaced $\langle 110\rangle$ antiphase domains.

2 (with a close up of a $\{100\}$ peak shown in the inset) shows a corresponding array of secondary peaks about each LAO diffraction peak (appearing like a four pointed star). These secondary or superlattice peaks are distributed along the $\{110\}$ direction and have a spacing of $0.060 \pm .002 \mathrm{~nm}^{-1}$ corresponding to real space repeat distances of $16.7 \pm 0.6 \mathrm{~nm}$. The kinematically forbidden $\{n 00\}$ and $\{0 n 0\}$ peaks (where $n$ is odd) are evident and all LAO peaks in this orientation

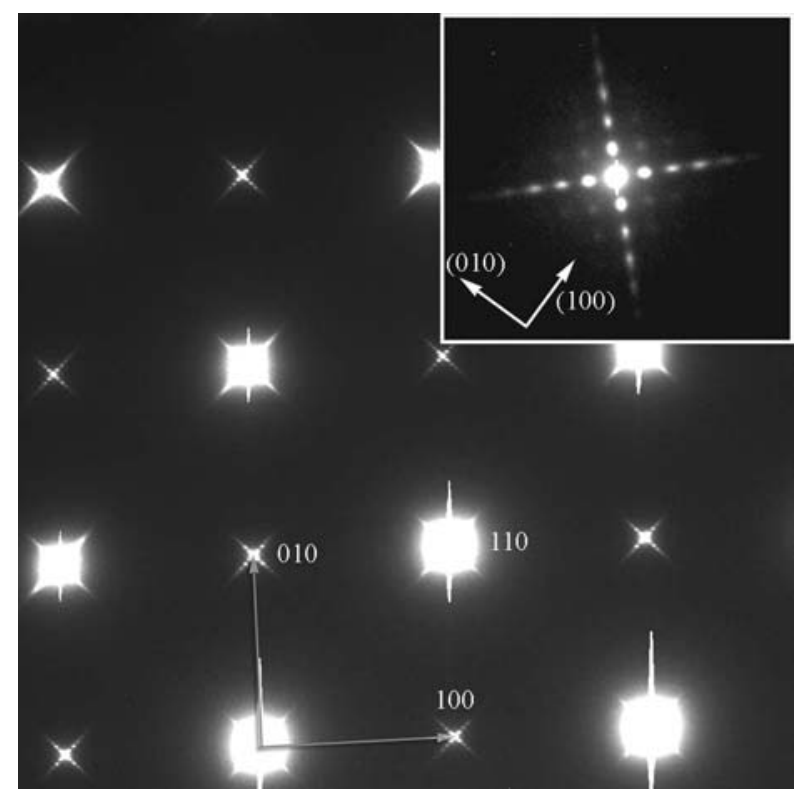

FIG. 2. Electron diffraction pattern of the $\gamma$ phase LAO in the [001] zone axis direction. The strong peaks are from the $\{110\}$ family and the weaker peaks from the $\{100\}$ family. The $\{100\}$ spacings are marked with arrows. All peaks show streaking (closely spaced satellite or superlattice peaks) in the $\{110\}$ directions. The additional vertical streaks on the brightest peaks are blooming in the CCD readout. The inset shows a close up of one peak with the distinct superlattice peaks clearly visible.

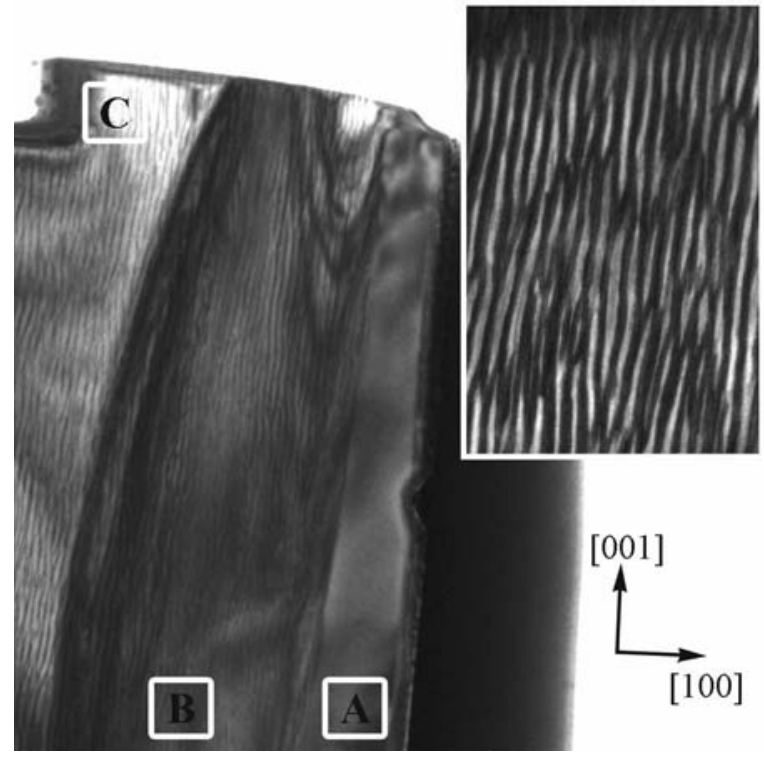

FIG. 3. Bright field TEM image in the [010] direction. This slice taken through and along a $\beta$ phase mesa. Inset shows a close up of typical bulk LAO in this orientation. Regions A, B, and C are the same as in Fig. 1.

show superlattice peaks with the primary peak position being the strongest. Looking in the [010] direction, the images (Fig. 3) look like a mass of strings or noodles stretched out along a common length [001], but intermixed perpendicular to that direction. The diffraction pattern here does not show any superlattice peaks, but does show a broadening in the (100) direction. Due to electron beam damage of the LAO, analysis was limited to low beam intensity TEM and diffraction studies.

\section{DISCUSSION OF BULK LAO}

LAO is a chemically ordered structure with possible variants of the relative lithium and aluminum positioning. We suggest an explanation of the observed lines and superlattice structures as arising from an antiphase domain structure in the chemical ordering.

Copper-gold $(\mathrm{Cu}-\mathrm{Au})$ is a typical antiphase domain prototype material. The ordered $\left(L 1_{0}\right.$ structure) face-centered cubic lattice has alternating planes of copper and gold. A given ordered domain may be offset by one layer from its neighbor domain. The result is a copper plane matched up with a gold plane and a resulting phase shift between the representation of the two regions. These are called antiphase boundaries and an antiphase domain structure. In the case of copper-gold ( $\mathrm{CuAu}$ II), the domains form a periodic structure with a repeat spacing of $4 \mathrm{~nm}$ (boundaries every $2 \mathrm{~nm}$ ). ${ }^{20,21}$ The resulting diffraction patterns show not only peaks associated with the chemical ordering (also called superlattice peaks) $[\{110\}$ and (001)], but the chemical ordered peaks are split by the antiphase domain ordering into satellite peaks. These satellite peaks are transferred to all allowed reflections by double diffraction. They indicate a long period modulation or superlattice in the material. The satellite peaks are what we refer to as superlattice peaks. In the bright field and dark field images, the fundamental and satellite superlattice peaks interfere (much like high resolution imaging or moiré 

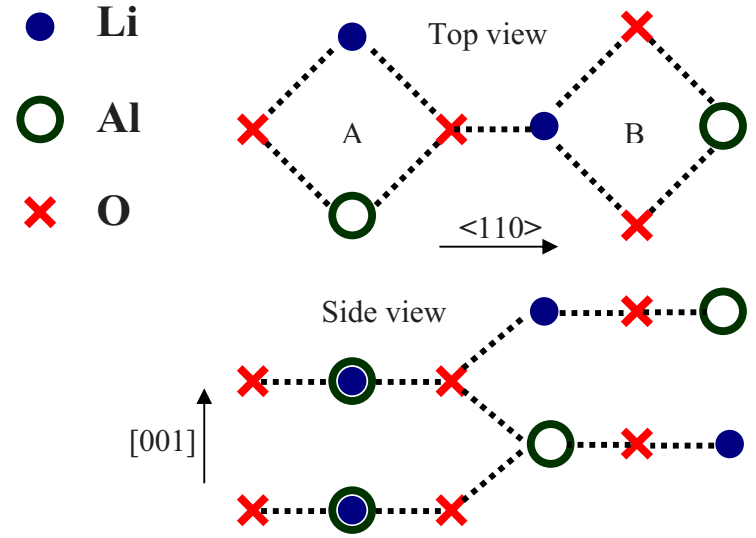

FIG. 4. (Color online) The LAO $\gamma$ phase model structure. The top view shows only the top two "squares" out of four. In lower layers, switch Li and $\mathrm{Al}$ as seen in side view. Labeled directions are relative to base unit cell. Pair $\mathrm{A}$ and $\mathrm{B}$ are marked.

fringes) and produce images with corresponding lines and cross hatching. In LAO, the spacing of the superlattice peaks and images indicates a larger spacing near $17 \mathrm{~nm}$.

LAO has a more complex structure than copper-gold, but like $\mathrm{Cu}-\mathrm{Au}$, has possible variants in its chemical ordering. Gamma phase lithium aluminate $(\gamma$-LAO) has a tetragonal lattice structure $\left(P 4{ }_{1}{ }_{1} 2\right)$ with lattice constants of $a$ $=0.5168 \mathrm{~nm}$ and $c=0.6268 \mathrm{~nm}$. This tetragonal representation is not the best way to see the ordering and possible phase variants. The basic LAO building block as shown in Fig. 4 is an approximate square of atoms: one aluminum, one lithium, and two oxygen. In this square the aluminum and lithium atoms are on opposite corners and the oxygen atoms on the other two corners. In the full structure, these are not perfect squares because the lithium to oxygen spacing is shorter than the aluminum to oxygen spacing and there are small shifts out of the plane for the oxygen atoms. However, it is nearly square and simplifies the following discussion. It takes four of these "square" building blocks to create a unit cell of LAO. Two of these squares are placed on top of each other with lithium above aluminum (and aluminum above lithium) and oxygen above oxygen (call this pair A). The other two (pair B) are similarly arranged (relative to each other), but rotated $90^{\circ}$ about the $c$ axis, offset in the $c$ direction by $\frac{1}{4}$ [001], and shifted sideways in the original [110] direction. This places each lithium (or aluminum) between four oxygen atoms; two in the plane with its square and the other two from offset "squares" above and below it.

A phase boundary (switching lithium and aluminum, or more accurately shifting one side of the boundary by $\frac{1}{2}$ [001] relative to the other side) in LAO is more complicated than in $\mathrm{Cu}-\mathrm{Au}$. A phase boundary that passed through at square would create squares with two lithium and two aluminum, which in our simplified model would seem acceptable. However, this is unacceptable in the real lattice where the different bond lengths would place the structure under considerable stress. A phase boundary placed between pairs A and B (or B and A) would have minimal impact. The lithium or aluminum sites in one square are separated from the lithium or aluminum sites in the next square by an oxygen atom, with that connecting oxygen atom bonding to an aluminum below, a lithium above, and a lithium and aluminum in the plane. A phase boundary, as far as that connecting oxygen is concerned, would just change the relative positions of lithium and aluminum on one side of the boundary, but the connecting atom would have the same set of nearest neighbor bonds and bond angles as those without a phase boundary. In the real lattice, there would be some small amount of stress associated with this type of boundary.

We suggest that the experimentally observed images and diffraction patterns can be explained with a simple $\frac{1}{2}$ [001] phase domain structure and double diffraction. Like $\mathrm{Cu}-\mathrm{Au}$, the phase domains are ordered. Because of the minimal effect from a phase boundary, the driving force for domain boundary ordering is weak and the spacings are large $(\sim 17 \mathrm{~nm})$. The two $\langle 110\rangle$ directions are equivalent and as expected superlattice peaks are seen in both equivalent directions. Electrons interact strongly with materials and the FIB prepared sample is sufficiently thick that many diffraction events occur. When many diffraction events occur, the expected diffraction pattern is the kinematical pattern convolved with itself. This results in all diffraction peaks showing superlattice spacings, even if only a subset of those peaks would show them by detailed diffraction considerations. The observations of the kinematically forbidden (100) peaks are evidence that many diffraction events are being observed. The (100) peak is forbidden in LAO, but the $\{120\}$ and $\{020\}$

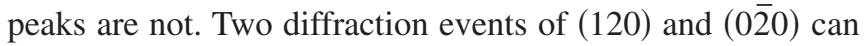
give rise to the observed (100) peak in this structure.

\section{WAFER SURFACES: SURFACE $\boldsymbol{\beta}$ PHASE}

Cut and polished $\gamma$ LAO wafers from an alternate supplier reacted differently from the previous batches to the typical $\mathrm{HCl}$ acid etch. Upon etch, the wafer surface was left opaque instead of clear. This is much like the description of Sun et al. ${ }^{18}$ where they mention visible roughening upon an $\mathrm{HCl}$ etch. Scanning electron microscope images showed rows or mesas or ridges running along the [001] direction of the etched $\gamma$ LAO wafer.

Diffraction analysis of these mesas showed a distinctly different crystal structure which we identified as the $\beta$ phase of lithium aluminate by both TEM diffraction analysis and SEM-based EBSD. This phase is present in the as received wafers, but the $\beta$ phase appears to have a different etch rate than the $\gamma$ phase. Thus, the $\beta$ phase leaves mesas upon etch and a multifaceted opaque surface. Figures 1 and 3 show typical TEM images looking into the [001] and [010] directions of the etched LAO wafer. Region A in those images shows the $\beta$ phase, $\mathrm{B}$ a transition region, and $\mathrm{C}$ the bulk $\gamma$ phase. The phase domains present in the $\gamma$ phase are absent in the $\beta$ phase. We thus identify the roughing of the surface as arising from a surface $\beta$ phase of lithium aluminate.

This surface $\beta$ phase, when seen in unetched wafers has a semicircular shape (when viewed in the $\gamma$-[001] direction, looking along the ridge or mesa) with the flat part of the semicircle at the wafer surface. The spacing along the surface between $\beta$ phase regions averages 1.5 to $2.0 \mu \mathrm{m}$ (center to center) with about $0.5 \mu \mathrm{m}$ of $\gamma$ phase between them. There are steps on the unetched surface, between the $\gamma$ and $\beta$ 


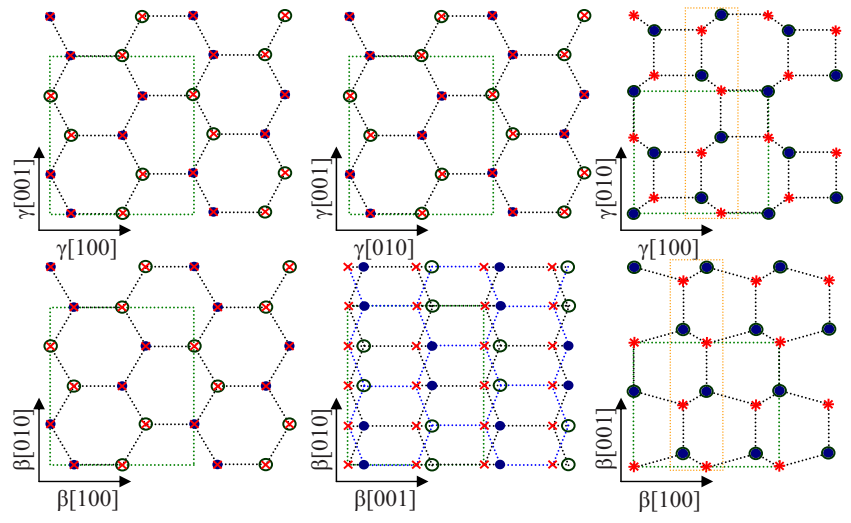

FIG. 5. (Color online) Crystal structures of the $\gamma$ (top) and $\beta$ phases (bottom). Aluminum atoms are hollow circles, lithium are solid blue circles, and oxygen atoms are red crosses. The unit cells in each view are shown. The top-bottom pairs match according to the experimentally observed relationship. In the $\beta$ [100] view, the blue lines show a surface that would match with the $\gamma$ phase. The atomic fit in the $\beta[100]$ and $\gamma[001]$ is poor. The column of atoms marked with a box would make a good match, but the neighboring column would require atomic rearrangements to fit.

phases, of approximately $20 \mathrm{~nm}$ with the $\beta$ phase being higher than the $\gamma$. Samples that underwent GaN growth show no $\beta$ phase, but do have steps on the surface of about $40 \mathrm{~nm}$ that are spaced consistent with the original $\beta$ phase spacing.

There is an epitaxial relationship between the $\gamma$ and $\beta$ phases. The $\beta$ phase is orthorhombic (Pna2 $2_{1}$ ) with lattice constants $a=0.528 \mathrm{~nm}, b=0.630 \mathrm{~nm}$, and $c=0.490 \mathrm{~nm}$. Experimentally, the $\beta-b$ axis aligns with the $\gamma-c$ axis, $\beta$ - $a$ axis aligns with the in-plane $\gamma-a$ axis, and the $\beta-c$ axis aligns with the out-of-plane $\gamma-a$ axis. This is consistent with the near match of lattice constant between the $\beta a$ and $b$ axes and the $\gamma a$ and $c$ axes. The in-plane lattice is a near match $(\sim 2 \%$ and $0.5 \%)$ and both structures show very similar atomic arrangements in that surface. The more significant mismatch $(\sim 4 \%)$ is in the out-of-plane direction which also has distinctly different atomic arrangements.

We tested the thermal stability of the $\beta$ phase with two experiments. An unetched wafer was placed in the GaN growth reactor, brought to growth temperature (but growth was not initiated), cooled, and then etched. This annealed wafer etched clear and smooth (like previous batches) indicating that the growth temperature was sufficient to reconvert the $\beta$ phase on the surface to the $\gamma$ phase of the bulk. Next, an opaque etched wafer was broken into several smaller pieces. Each piece was annealed in an inert gas to a given temperature for $30 \mathrm{~min}$. TEM samples were prepared from each annealed piece and the presence or absence of the $\beta$ phase in the etched mesas was checked. We found that at $450{ }^{\circ} \mathrm{C}$ or below, the $\beta$ phase was still present, but at $500{ }^{\circ} \mathrm{C}$ or above, the mesa regions had converted to typical $\gamma$ phase material.

\section{DISCUSSION OF $\beta$ PHASE}

Figure 5 shows the atom arrangements in the three perpendicular directions of the two phases of LAO. The matching of the top $(\gamma)$ and bottom $(\beta)$ pairs is based on the experimentally observed relationships. These views are somewhat idealized, like in the previous discussion of the $\gamma$ phase. In the $\gamma$ [010] out-of-plane view (left pair) there is a near identical match of atomic positions. In the $\beta$ [100] (middle pair) view, we see that there is a plane of atoms (the set marked in blue) that make a good fit to the $\gamma[100]$ arrangement. That matching plane is marked in the $\beta[010]-\gamma$ [001] views (right pair) as a column. The columns to either side do not make a good match and would require significant atom movement to have those two atomic faces fit together. However, experimentally we see that this $(\gamma[001]$ and $\beta$ $[010]$ ) is the long direction of the $\beta$ phase ridges and very little of this match would be required.

Region B, just below the surface phase (region A) in Figs. 1 and 3, shows substantial diffraction contrast. This is presumably due to the strain or lattice distortion put on the $\gamma$ phase material by the mismatch with the $\beta$ phase. We suggest that this strain limits the size and shape of the $\beta$ phase regions. The lattice mismatch between these two materials is greatest in the out-of-plane direction (4\%), intermediate in the in-plane $\gamma-a$ direction (2\%), and minimal in the $\gamma-c$ direction $(0.5 \%)$. If we imagine a $\beta$ phase seed region epitaxially growing within the $\gamma$ phase material, we see that the build up of strain would become significant. However, the strain build up would depend upon and affect the growth directions. The $\beta$ phase would appear to be energetically more stable at the surface than the $\gamma$ phase, but can only grow until the strain energy cost balances the energy gained by the conversion to $\beta$ phase. In the in-plane $\gamma$ - $a$, direction the growing $\beta$ phase seed would be strained by an $\sim 2 \%$ mismatch, while in the $\gamma-c$ direction, the lattice match is closer $(\sim 0.5 \%)$ and there is little barrier to propagation of the $\beta$ phase in that direction. In the out-of-plane direction, there is a free surface that can absorb some of the larger $(\sim 4 \%)$ mismatch, but as the $\beta$ phase seed grows in depth, that mismatch would also have a strain limiting role. Thus, the $\beta$ phase will strain limit in the $\gamma-a$ directions and not in the $\gamma-c$ direction, thereby producing a structure with a long axis along the $\gamma-c$ direction and short dimensions along the $\gamma-a$ as observed. This self-limitation on growth would have some possible variations (spacing and depth for instance) arising from the wafer conditions (temperature, smoothness, etc.) when the $\beta$ phase forms and grows.

This beta phase morphology is consistent with features observed by others on LAO wafers and in thin film GaN growth on those wafers. The corregated or "slate" morphology of GaN thin films on LAO directly mirrors the surface morphology seen on the LAO. Sun et al. ${ }^{11,18}$ showed AFM micrographs of this surface morphology as-received and after a $750{ }^{\circ} \mathrm{C}$ anneal. Their wafers showed ridges spaced about $1.0 \mu \mathrm{m}$ center to center with height changes of up to 5 $\mathrm{nm}$ in the as-received wafers and $15 \mathrm{~nm}$ in the annealed wafers. Our wafers showed similar $\beta$ phase spacing (1.5 to $2.0 \mu \mathrm{m})$ and similar surface steps $(20 \mathrm{~nm}$ before growth and $40 \mathrm{~nm}$ after). We identify the commonly observed LAO surface morphology and GaN morphology as arising from this surface $\beta$ phase.

The $\beta$ phase has a higher density than the $\gamma$ phase. When the $\beta$ phase is converted back to the $\gamma$ phase, there must be a volume increase. Sun et al. ${ }^{11,18}$ observed an enhancement in LAO corrugation after annealing. They suggested that this 
enhancement was due to decomposition of the LAO. While the structure may decompose, in light of the observed $\beta$ phase, an alternate explanation of their observation is the volume increase from the $\beta$ to $\gamma$ reconversion.

It would appear that the $\beta$ phase is thermodynamically more stable than the $\gamma$ phase at room temperature. However, the apparent lack of significant $\beta$ phase on some wafers makes it difficult to attribute an origin or formation time to this feature and suggests further study is needed. As the $\beta$ phase appears only on the surface of each wafer, it must be forming after or during the final stages of polishing. It is interesting that wafers from one cut-polish supplier showed the surface phase and another did not, suggesting that it is either generated by the processing or removed by some final step in the processing. The observations that the $\gamma$ phase surface is below the level of the $\beta$ phase surface in the asreceived wafers suggest that some processing step (mild etch or final polish) has taken place with the $\beta$ phase in place. Variations in that final processing step would explain differences in the observed step heights. It is clear from the literature that the $\beta$ phase is an extremely common feature of LAO wafers and has impact on the usefulness of LAO as a GaN substrate.

Heating to the growth temperature is sufficient to convert the $\beta$ to $\gamma$ phase, but in the commercially available wafers it is not sufficient to remove all memory of surface phase from the final product. The surface $\beta$ phase can be controlled (removed) by simple $500{ }^{\circ} \mathrm{C}$ anneals. We suggest performing anneals prior to any etch or preferably, even before any final polishing or processing steps.

\section{CONCLUSION}

We have seen that $\gamma$ lithium aluminate (a near lattice matched substrate for $\mathrm{GaN}$ ) forms antiphase domains with a very long periodic spacing of $16.7 \pm 0.6 \mathrm{~nm}$ in the $\langle 110\rangle$ direction. These domains are columnar and large in the [001] direction. Depending upon wafer processing, a surface phase of $\beta$ lithium aluminate commonly forms on the $\gamma$ LAO wafer and is the origin of the commonly observed slatelike or cor- rugated surface morphology. The $\beta$ phase size and shape appear to be strain limited within the larger $\gamma$ phase and will revert to the $\gamma$ phase at $500{ }^{\circ} \mathrm{C}$ or above. It aligns with the $\gamma$ phase: $\beta-b$ to $\gamma-c$ axis, $\beta-a$ to in-plane $\gamma-a$, and $\beta-c$ with out-of-plane $\gamma$ - $a$.

${ }^{1}$ M. Marezio, Acta Crystallogr. 19, 396 (1965).

${ }^{2}$ E. F. Bertaut, A. Delapalme, G. Bassi, A. Durif-Varambon, and J.-C. Joubert, Bull. Soc. Fr. Mineral. Cristallogr. 88, 103 (1965).

${ }^{3}$ B. Cockayne and B. Lent, J. Cryst. Growth 54, 546 (1981).

${ }^{4}$ E. S. Hellman, Z. Liliental-Weber, and D. N. E. Buchanan, MRS Internet J. Nitride Semicond. Res. 2, 30 (1997).

${ }^{5}$ K. Xu, J. Xu, P. Deng, R. Qiu, and Z. Fang, Phys. Status Solidi A 176, 589 (1999).

${ }^{6}$ P. Waltereit, O. Brandt, and K. H. Ploog, Appl. Phys. Lett. 75, 2029 (1999).

${ }^{7}$ P. Waltereit, O. Brandt, A. Trampert, H. T. Grahn, J. Menniger, M. Ramsteiner, M. Reiche, and K. H. Ploog, Nature (London) 406, 865 (2000).

${ }^{8}$ H. P. Maruska, D. W. Hill, M. C. Chou, J. J. Gallagher, and B. H. Chai, Opto-Electron. Rev. 11, 7 (2003).

${ }^{9}$ R. R. Vanfleet, J. A. Simmons, H. P. Maruska, D. W. Hill, M. M. C. Chou, and B. H. Chai, Appl. Phys. Lett. 83, 1139 (2003).

${ }^{10} \mathrm{P}$. Waltereit, O. Brandt, M. Ramsteiner, R. Uecker, P. Reiche, and K. H. Ploog, J. Cryst. Growth 218, 143 (2000).

${ }^{11}$ Y. J. Sun, O. Brandt, and K. H. Ploog, J. Vac. Sci. Technol. B 21, 1350 (2003).

${ }^{12}$ B. A. Haskell, A. Chakraborty, F. Wu, H. Sasano, P. T. Fini, S. P. Denbaars, J. S. Speck, and S. Nakamura, J. Electron. Mater. 34, 357 (2005).

${ }^{13}$ C. Q. Chen, M. E. Gaevski, W. H. Sun, E. Kuokstis, J. P. Zhang, R. S. Q. Fareed, H. M. Wang, J. W. Yang, G. Simin, M. A. Khan, H.-P. Maruska, D. W. Hill, M. C. Chou, and B. Chai, Appl. Phys. Lett. 81, 3194 (2002).

${ }^{14}$ M. D. Reed, O. M. Kryliouk, M. A. Mastro, and T. J. Anderson, J. Cryst. Growth 274, 14 (2005).

${ }^{15}$ A. Mogilatenko, W. Neumann, E. Richter, M. Weyers, B. Velickov, and R. Uecker, J. Appl. Phys. 102, 023519 (2007).

${ }^{16}$ J. W. Gerlach, A. Hofmann, T. Hoche, F. Frost, B. Rauschenbach, and G. Benndorf, Appl. Phys. Lett. 88, 011902 (2006).

${ }^{17}$ J. Zou, S. Zhou, J. Xu, X. Zhang, X. Li, Z. Xie, P. Han, and R. Zhang, J. Mater. Sci. 41, 5937 (2006).

${ }^{18}$ Y. J. Sun, O. Brandt, U. Jahn, T. Y. Liu, A. Trampert, S. Cronenberg, S. Dhar, and K. H. Ploog, J. Appl. Phys. 92, 5714 (2002).

${ }^{19}$ M. M. C. Chou, H. C. Huang, D.-S. Gan, and C. W. C. Hsu, J. Cryst. Growth 291, 485 (2006).

${ }^{20}$ P. Hirsch, A. Howie, R. Nicholson, D. W. Pashley, and M. J. Whelan, Electron Microscopy of Thin Crystals (Krieger, Malabar, FL, 1977), pp. 378-387.

${ }^{21}$ J. M. Cowley, Diffraction Physics (Elsevier, Amsterdam, 1995), pp. 405407. 